\title{
Relevance of a standard food model in combination with electronic jaw movement recording on human mastication pattern analysis
}

\author{
Gregor Slavicek $^{1 *}$, Mikhael Soykher ${ }^{2}$, Marina Soykher $^{2}$, Haymo Gruber $^{3}$, Peter Siegl $^{4}$
}

${ }^{1}$ Steinbeis Transfer Institut Biotechnology in Interdisciplinary Dentistry der Steinbeis Hochschule Berlin, Stuttgart, Germany;
${ }^{2}$ Institute Biotechnology in Interdisciplinary Dentistry, Moscow, Russia;
${ }^{3}$ Dental University of Graz, Austria, and FH Joanneum, Austria, Private Practice, Stallhofen, Austria;
${ }^{4}$ Private Practice, Weiz, Austria.
Email: gregor.slavicek@stw.de

Received 13 February 2010; revised 7 March 2010; accepted 9 March 2010.

\section{ABSTRACT}

The aim of this paper is to describe the possibilities of analyzing human mastication. The development of a standardized food model is presented. Based on the findings of a systematic literature search an elastic food model was created with the aim of standardizing size and elastic properties. Three different eatable jellied products were chosen, created by a changing of the amount of gelatin (260 Blooms) related to the total mass of the standard jellied food. The different hardness were classified in soft, medium and hard, flavored identically, but stained with different colors: soft-green, medium-yellow, hard-red. A cylindrical form was chosen with a height of $1 \mathrm{~cm}$ and a diameter of 2 cm. A standard protocol for analyzing chewing patterns in men was created. The condylographic data off several patients are described in details to demonstrate the possible clinical implementation. The newly developed standard food model (SFM) showed the capability to serve in experimental settings to analyze human mastication, although only a few patients have been examined. In addition, strength and size of the newly developed SFM load the masticatory system in an extent; it should be possible to disclose subclinical symptoms of patients within a short time of examination. The diagnostic procedure of temporo-mandibular disorder (TMD) should be endorsed by this new method. The condylographic data created by a standardized protocol should have the ability to enhance the clinical functional analysis of patients previous to restorative dental procedures.

Keywords: Human Mastication; Standard Food Model;
Mandibular Movement; EMG; Condylography; Biomechanics; Temporo-Mandibular Joint; Swallowing; Chewing; Chewing Muscle Activity; Craniomandibular System; Nutrition; Tooth Loss

\section{INTRODUCTION}

Mastication is an essential part of the digestive process. The ability of the masticatory organ to mince and process food is directly related to with the individual's vital functions and general state of health. Loss of teeth and the reduced ability to chew have a significant impact on an individual's nutritional condition, health, gastrointestinal disorders, and digestion [1]. However, confirmed evidence of these associations is scarcer than one would expect it to be: key co-factors and variables are yet unknown or only partially identified. In the existing studies, the nutritional habits of the study participants have been poorly or fragmentarily documented. Extrapolation of data from animal experiments is such that the results may be poorly applicable to the human setting [1]. Lifestyle, morbidity and the individual's socioeconomic status are major factors influencing nutrition and oral health.

If one views prosthetic and restorative measures in the light of nutrition and diet, it would be meaningful to explore dental measures critically. In a large and systematic overview about nutrition and its relationship with oral health and systemic disease, the authors showed that the negative impact of edentulism on nutritional habits are inadequately compensated for by dental prostheses and restorative measures [2]. Furthermore, a reduced number of teeth may cause changes in weight; the age and characteristics of the respective population are also involved in this phenomenon. New and well structured studies are needed to evaluate the association between 
nutrition, dental status and the individual's state of health. Prospective longitudinal studies taking relevant factors such as mastication and occlusion into account would be consequential [2]. A hypothetical model of the associations between oral health and nutrition is shown in Figure 1.

Patients without dementia have twenty-fold more teeth in their mouth than age-matched patients with severe Alzheimer dementia (4.7 vs. 0.2 teeth per patient). The results of the Neuburg dementia study were highly significant. The presence of a large number of teeth in advanced age was identified as a protective factor in respect of Alzheimer dementia. Conversely, edentulism was an independent risk factor for Alzheimer dementia, but not for vascular dementia or ischemic stroke [3].

Occlusion, occlusal interferences, and the severity of craniomandibular disorders (CMD) appear to be associated with mastication: the more numerous the occlusal interferences, the more advanced the CMD, the longer the individual takes to masticate a specific type of food and the more pronounced are the person's atypical masticatory movements [4]. These results may be interpreted as follows: compensatory masticatory patterns occur on a reflex basis, but the effectiveness of such masticatory patterns is doubtful and it may be presumed that these patterns accelerate the progression of a CMD [4].

Human mastication is controlled by the central nervous system. Properties such as the hardness, plasticity, elasticity and size of the ingested food directly influence the feedback control system. Age, gender, and dental status are intrinsic modulators. All of these factors may influence the sequence of mastication. Vertical and lateral amplitudes as well as the velocity of jaw movements during mastication are parameters that can be used for a targeted analysis of determinants that are expressive of the adaptation abilities of healthy individuals and volunteers [5]. On the other hand, the impact of age may not be as significant as was initially assumed. The number of

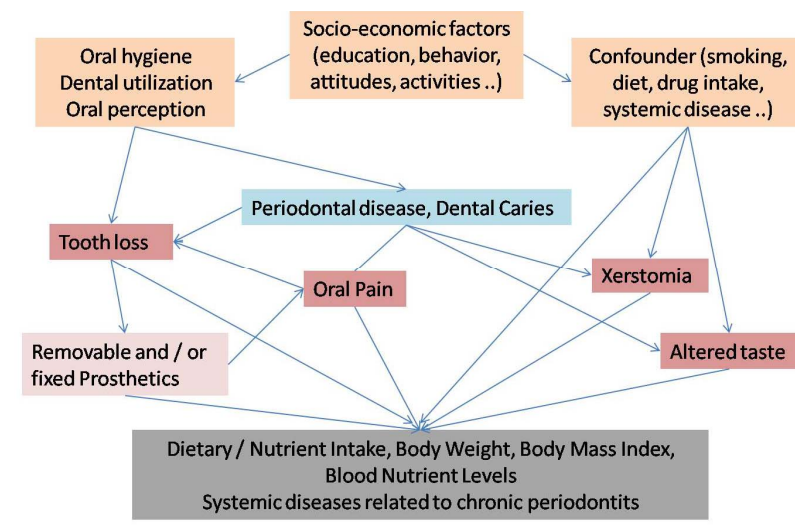

Figure 1. A hypothetical model demonstrating the complex interactions of oral health status, nutrition and systemic diseases (adapted from 17). masticatory cycles needed to grind a standardized unit of food increases with age, but the ability to adapt oneself to the properties of food is retained even by elderly individuals [6].

Muscular activity and the frequency of masticatory cycles may be influenced by the hardness of food in the initial stage of mastication [7]. Flow properties and the plastic behavior of food influence the entire masticatory cycle. This initial phase is controlled by a programmed pattern steered by the cortex and the brain stem, which adjusts the initial masticatory cycles to the food introduced into the mouth. A mechanism with sensory feedback from the structures of the stomatognathic system is located in the brain stem. This mechanism then takes over the function of modifying the muscle strength of the following masticatory movements to the quality of the ingested food [7].

Food is minced during mastication by a process of selection and breakup. The chances of a component of food being "selected" between the teeth may be viewed in relation to the size of the individual unit of food. The actual breakup of food may be described as a cumulative dividing function. Computer simulations based on these two processes could be used to describe the individual's efficiency of mastication [8]. The efficiency of mincing food may also be determined with the aid of filter methods. A functional model based on a model of human mastication describes the qualitative abilities of the masticatory organ.

Mastication and brain activity are closely interrelated. With the aid of functional magnetic resonance imaging (fMRI) it was shown that mastication involves a bilateral increase in the activity of the sensomotor cortex, the thalamus, the amygdala and the cerebellum [9]. In elderly persons, the magnitude of the increase was less in the first three above mentioned regions, but greater in the cerebellum. Therefore, in the elderly the function of mastication is integrated in the neuronal circuits of the hippocampus and appears to play an important role in preventing age-related disorientation. Mastication may be a useful therapy to prevent senile dementia [9]. Human mastication is supported to a great extent by the tongue. Positioning the food, exploring its consistency and size, as well as aiding the process of changing sides, are essential functions without which mastication would be impossible. Bilateral mastication of chewing gum modulates the activity of the primary sensomotor cortex during tongue movements, as determined on fMRI investigations. This activation appears to differ in the hemispheres, depending on the preferred side of mastication. These data support the postulated existence of short-term memory functions which store the recently executed movement pattern. Stimulation of the brain in the elderly or rehabilitation of injured brains can be supported by controlled mastication activity [9]. 
Mastication is a concrete load for the mandible and the craniomandibular joint. Loads on the mandible during the closing action reduce the intraarticular distance in the craniomandibular joint [10]. Ipsilateral craniomandibular joints are loaded less during mastication than contra-lateral joints [11].

Forces acting on the jaw during masticatory movement have been investigated. It was found that maximal forces are achieved in the vicinity of the intercuspal position. The forces are lowest when the jaw is opened [12]. The consistency of food is a major factor that modulates muscle strength: harder food reduces closing strength during mastication, but this is not the case when soft food is chewed. The latter causes no significant changes. Persons who wear full prostheses have lost their ability to react adequately to the quality of food [12].

Electromyographic studies are used to analyze the masticatory muscles. A hypothetical model was used: simultaneous activation of the jaw openers during mastication leads to additional activation of the muscles involved in closing the jaw [13]. During mastication, additional and simultaneous activation of the digastric muscle was much more pronounced during closure than it is in isometric tension. However, such activation was mild compared to the maximum possible activation potential of the digastric muscle. Therefore, it may be concluded that simultaneous activation of the openers has no more than a mild impact on the additional activation of masticatory muscles [13]. It was also found that muscle activity measured with EMG does not always permit consistent conclusions about masticatory strength [14]. The higher muscle activity during mastication, as seen on EMG, is not directly associated with an increase in muscle strength-as compared to the activity of bruxism. Additional EMG measurements are needed to analyze the actual masticatory strength. It would not be permissible to draw conclusions about loads on the craniomandibular joints on the basis of muscle activity on EMG [14]. The hardness or consistency of food modulates EMG activity, especially during the initial masticatory movements [15].

Masticatory patterns seem to differ from person to person, and appear to be markedly dependent on intraoral and neuromuscular sensory feedback controls. A high reproducibility may be presumed, provided the chewed object has similar or identical properties [5]. However, it was shown that even healthy probands with class 1 occlusion and no major deviations or missing teeth, and no signs or symptoms of a functional disorder, vary to a certain extent even under standardized conditions [5]. The frequency of mastication, the speed of shift, the amplitude of motions, and the duration of closure were found to differ. Psychological and physical variables such as emotional status, irritation or fatigue may play a role in this context, but an explicit statement in this regard cannot be made yet [5].

Registration of masticatory movements by the use of electronic systems provides deeper insights into the motion and dynamics of the mandible and, at least indirectly, into movements of the craniomandibular joint. Mastication is characterized by gliding of the teeth, a pause in intercuspal position or near-intercuspal position, and wide lateral movements [16]. This is true for children as well as adults. Masticatory movements in the occlusion of milk teeth are marked by a pronounced lateral motion during opening. The extent of lateral movement during opening is typically larger than that during closure. In deciduous teeth, the extent of lateral movement reduces when opening while lateral excursion increases during closure. The adult reveals an entirely different masticatory pattern: medial opening and wide lateral closure characterize the adult masticatory pattern [16]. The properties of food influence masticatory cycles not only in adults but also in children. Hard food enhances the lateral component in the adult, and increases the medial one in children. Neither in the adult nor in the child does one find a pure hinge movement during mastication [16]. Separation of the molars on the working side is closely related to the size of the food bolus. This corroborates the hypothesis that opening does not occur incidentally during mastication, but is controlled by various feedback systems in order to minimize effort during mastication [17].

Swallowing and mastication are closely related to each other. The production of saliva is a basic prerequisite for successful mastication. However, the additional intake of fluid during a masticatory cycle influences mastication positively only when the individual ingests very dry food (for instance, cake); this is not the case when fatty (such as cheese) or watery foods (such as carrots) are ingested [18]. The optimal time point for mastication was investigated, and an optimized mastication and deglutition model was developed [17]. Interestingly, the number of masticatory cycles until the first act of swallowing was very constant in the individual proband, independent of the type of food, such as nuts compared to carrots. In fact, the number of masticatory cycles until the first act of deglutition was strongly correlated with the quantity of produced saliva [17].

Analysis of human mastication is an extremely functional resource of assessing the effectiveness of occlusion and the functional statfus of the stomatognathic system prior to a restorative or prosthetic intervention. For this purpose, it is necessary to standardize the investigation and the registration procedure of mastication. This article describes the use of a standardized model of food in combination with mandibular movement recordings. Principles of standard model food and standard procedures of chewing recording are described elsewhere [19-22]. 


\section{MATERIAL AND METHOD}

The model food used to analyze mastication should be elastic, possess different degrees of hardness, and be of a minimum size. Based on a conventional mass of gum for manufacturing commercial fruit gum, a standard cylindrical form (height $1 \mathrm{~cm}$, diameter $2 \mathrm{~cm}$ ) in three different degrees of hardness (soft, hard, medium) was produced (A. Egger' Sohn, Süsswaren und Naturmittel GmbH., Mellergasse 4, A-1230 Vienna, Austria). The degrees of hardness were achieved by adding different quantities of gelatin (edible gelatin SPM 5765, Biogel AG Haldenstrasse 11, CH-6006 Lucerne) to the ground mass (soft: $15.5 \mathrm{~g}$ per mass; medium: $23 \mathrm{~g}$ per mass; hard: $31 \mathrm{~g}$ per mass). (Figure 2(a) and 2(b)).

Masticatory movements are visualized by the use of a paraocclusal clutch and joint path registration. The paraocclusal clutch influences the shearing action of the teeth due to its buccal fixation. However, currently we have no other means of fixing the registration arches. The recordings described in this article were made by the use of the CADIAX diagnostic system ( $\mathbb{B}$ GAMMA med. wiss. FortbildungsGmbH, Josef Brennerstr. 10, A-3400 Klosterneuburg, Austria). All recordings had to be based on a standardized protocol in order to perform an optimal analysis and make inter-individual comparisons.

The analysis is started by performing a conventional visualization of the joint path in three planes (sagittal, frontal, transverse). Initially the extent (quantity) of motion is of interest. The quantity is most easily registered in the sagittal view. This can be determined for the working side (AS) and the non-working side (nAS). AS should be less in terms of quantity than nAS (Figure 3). The position of masticatory movements is then analyzed
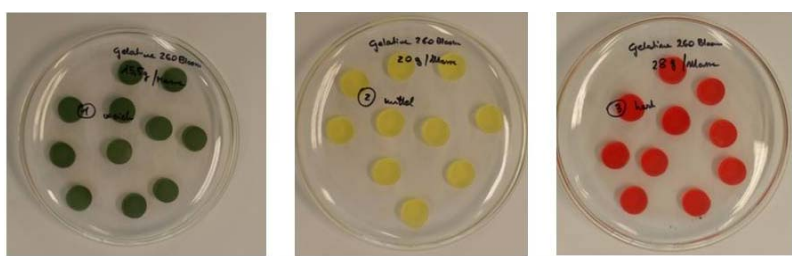

(a)
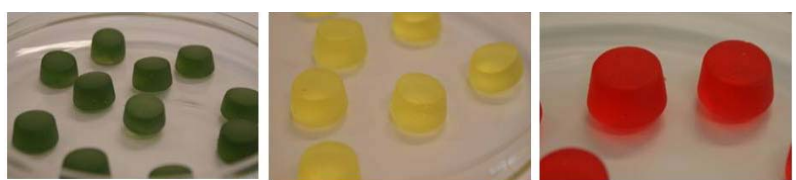

(b)

Figure 2. (a) Prototypes of the standard food model, green (soft) yellow (medium) and red (hard). The amount of gelatin (260 bloom) per mass was increased for the yellow and hard standard food model, to increase the differences of the elastic qualities; (b) prototypes of the standard food model, green (soft) yellow (medium) and red (hard) with a cylindrical form and the dimensions height $1 \mathrm{~cm}$ and diameter $2 \mathrm{~cm}$. in relation to standard movements (protrusion/retrusion, opening/closing, mediotrusion on the right and the left side). Movements coincident, below or above the standard motions are tolerable (Figure 4). In this context one

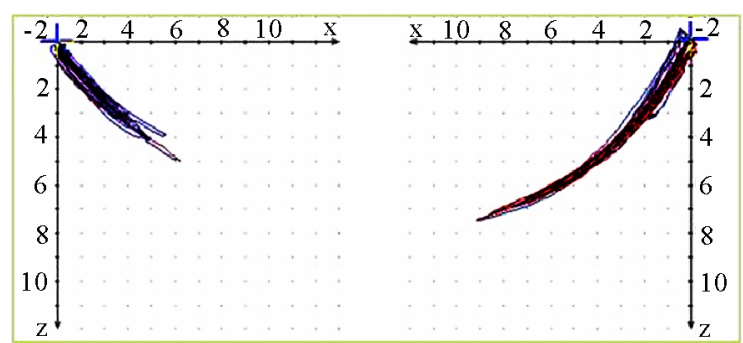

(a)

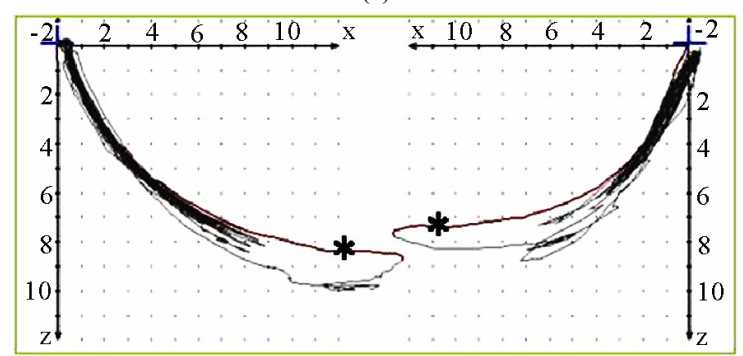

(b)

Figure 3. Sagittal view of recorded mastication pattern, (a) $21 \mathrm{y},+$, r, s; the right side is the ws and shows clearly a lesser quantity then the nws; (b) $49 y, \widehat{O}, 1, \mathrm{~s}$; the left side (ws) shows a lesser quantity then the nws. The wide movement $(*)$ indicates with the initial first opening for intake of the standard food.
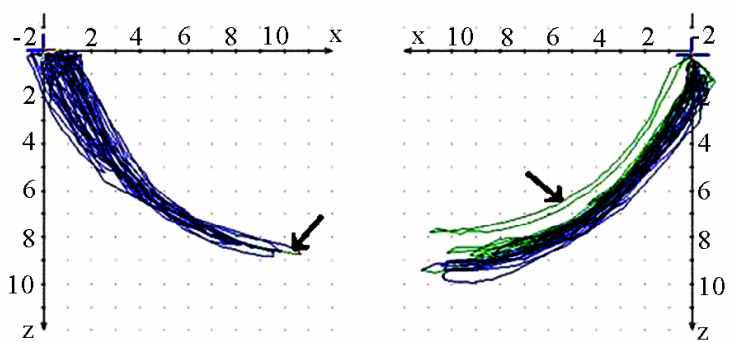

(a)
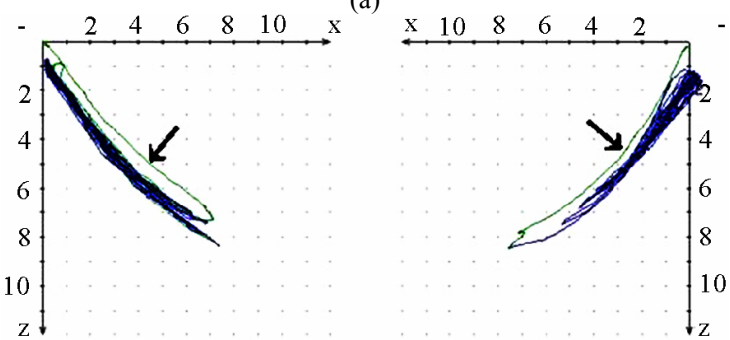

(b)

Figure 4. Sagittal view of recorded mastication pattern, (a) $18 \mathrm{y}, \mathrm{O}, \mathrm{r}, \mathrm{h}$; the right side is the ws, the standard movement (pro-re) is overlaid by the chewing cycles, the left side functions below pro-re; (b) $51 \mathrm{y}, \hat{\gamma}, \mathrm{r}, \mathrm{s}$; both sides sketches below the pro $(\swarrow)$ and are coincident with re. 
should also analyze whether the AS condyle is deviated in a different direction than the nAS condyle during mastication (Figure 5). As every movement is started from the reference position (RP) after renewed fixation, the position of masticatory movement can be assessed in relation to RP. Involvement or avoidance of this position may also be included in the analysis. Of course, AS and nAS can and should be assessed as well (Figure 6). Assessment of harmony can be achieved by the dynamic movement and by viewing the registered lines during the 18-second recording procedure (Figure 7).

Deviations from the points of occlusion (e.g., molar cusps) towards the sides are a physiological phenomenon during mastication and have been described in the published literature $[5,6]$. One should always consider the fact that the motion is a compound one. The movement of the mandible to the side and the lateral shift of the hinge axis can be analyzed in the frontal view during the motion (Figure 8).

Viewing the joint path registration from the cranial aspect (coronary view) provides information about the transverse component of masticatory movement. Movements towards one side or both sides are possible. The extent of motion should be noted. Large individual differences may be found. Associations between the quality of occlusion and the functional state of the ligaments of the stomatognathic system can be derived from these registrations (Figure 9).

Visualization of time-pathway diagrams enables the investigator to divide the masticatory cycles of AS and

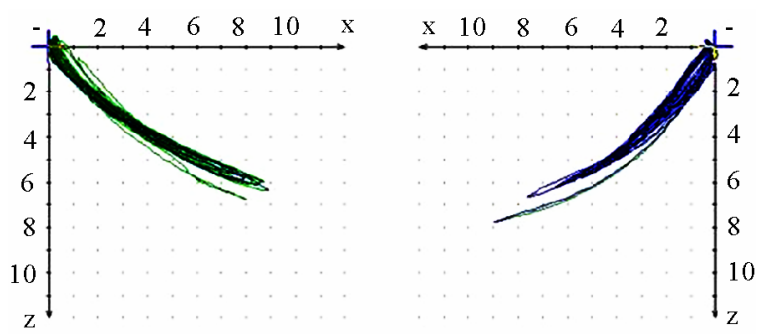

(a)
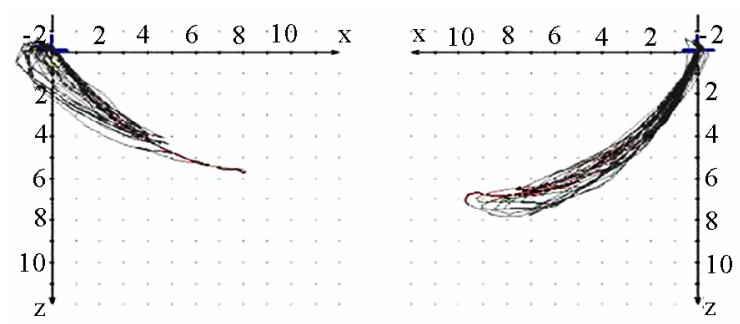

(b)

Figure 5. Sagittal view of recorded mastication pattern, (a) $21 \mathrm{y},+1,1, \mathrm{~s}$; the right side is the ws, pro-re of both sides are overlaid by the chewing cycles only during the initial phase of the mastication; (b) $17 \mathrm{y}, \circ, \mathrm{r}, \mathrm{h}$; pro-re is overlaid by the chewing cycles on both sides.
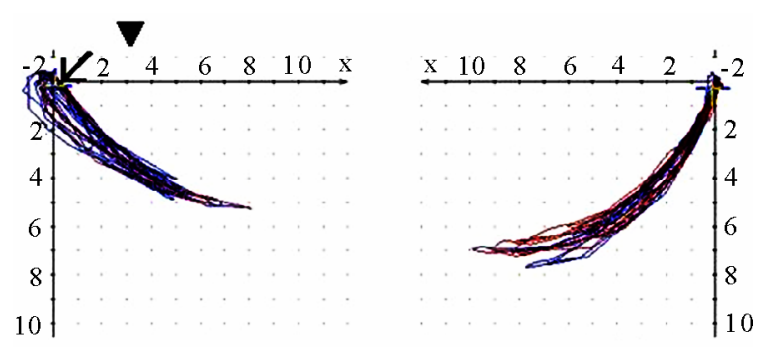

(a)
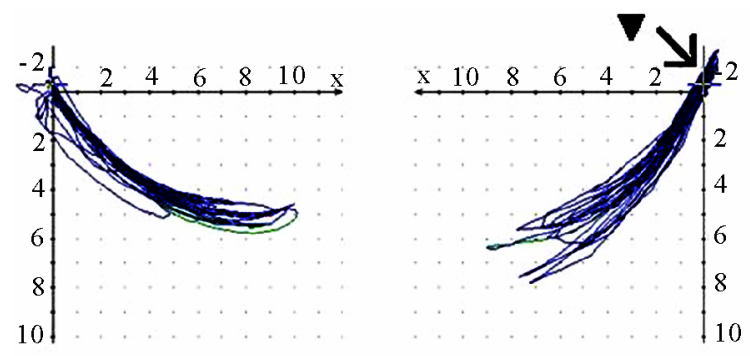

(b)
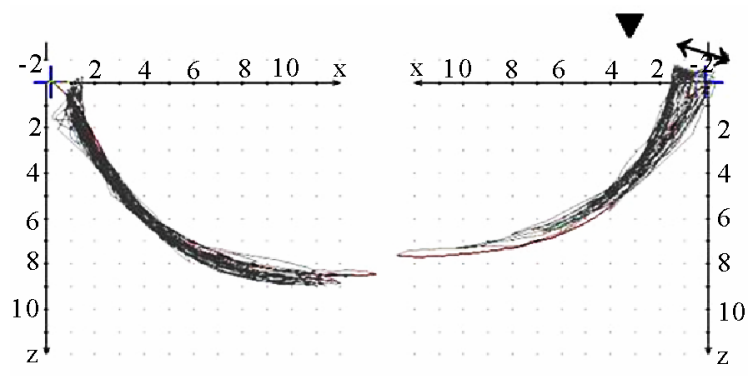

(c)
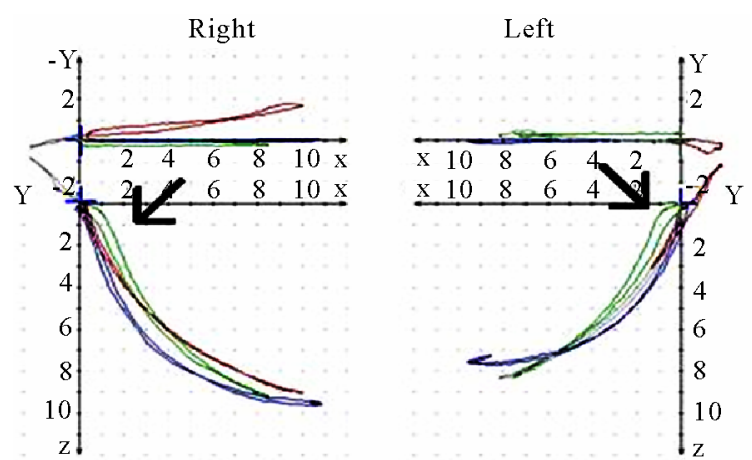

(d)

Figure 6. Sagittal view of recorded mastication pattern, (a) $17 \mathrm{y}$,, r, m; ws $(\boldsymbol{\nabla})$ shows a tracing behind RP $(\swarrow)$ and z-axis (vertical axis); (b) same participant as shown in 6(a), 1, s; ws ( $\nabla$ ) charts a Re-Sur-trusiv tracting $(\searrow)$; (c) $18 \mathrm{y}, \hat{O}, 1$, h; ws $(\boldsymbol{\nabla})$ uses the area in front and above RP $(\longleftrightarrow \longrightarrow)$ : participant with dental class $11 / 1$ and initial convexity in pro-re recording (see 6(d)); (d) same participant as shown in 6(c); standard tracings: pro-re: green, Open-Close (blue), Mediotrusion right (red), Mediotrusion left (grey). The initial convexity is indicated with arrows $(\swarrow)$. 

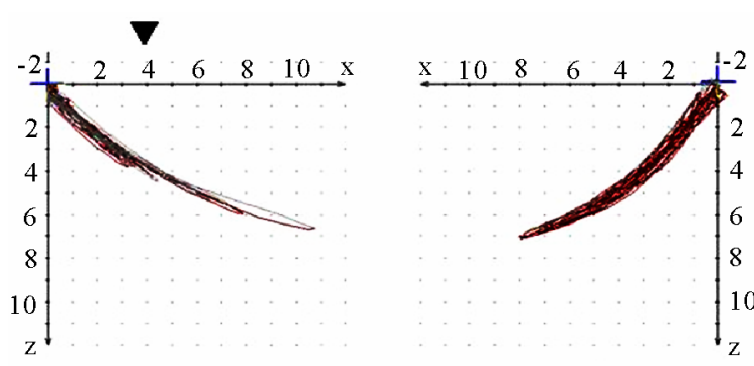

(a)
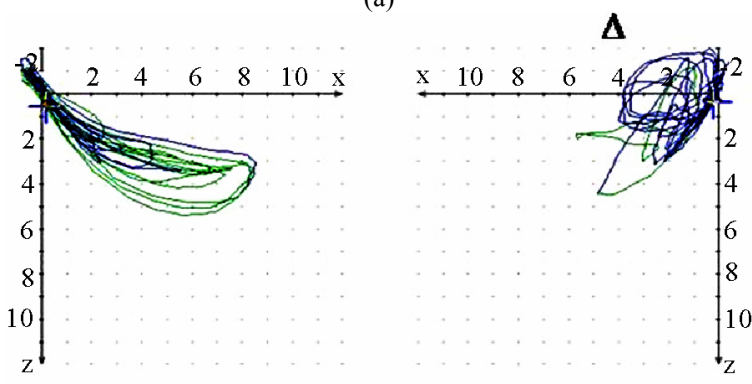

(b)

Figure 7. Sagittal view of recorded mastication pattern; (a) $21 \mathrm{y}$,, r, s; both sides (ws $\boldsymbol{\nabla}$ ) demonstrate a appropriate harmony of the chewing strokes; (b) $52 \mathrm{y}$, o, r, s; the ws displace a breakup of the harmony, nws $(\triangle)$ plots a non harmonic chewing pattern.
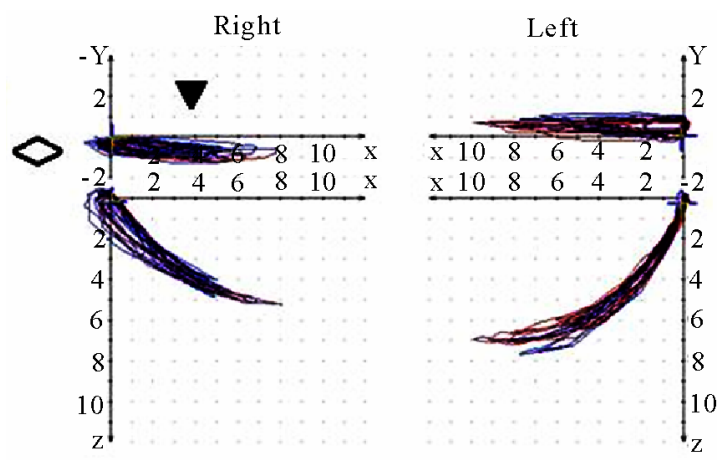

(a)
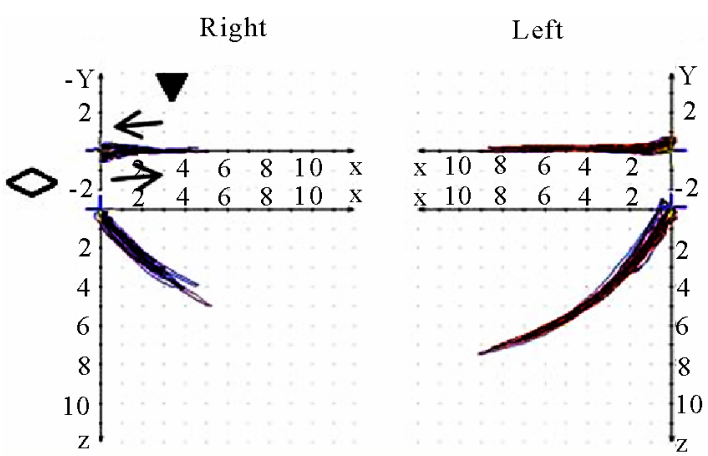

(c)

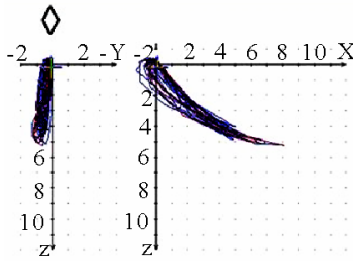

(a)

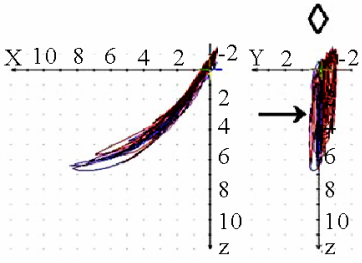

(b)

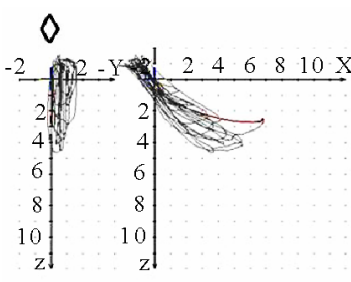

(c)

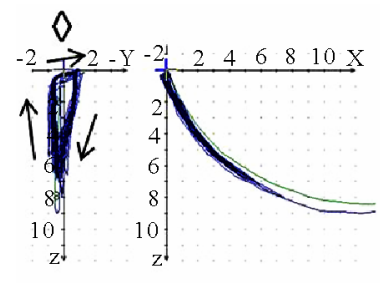

(d)
Figure 8. Frontal view $(\diamond)$ of recorded mastication pattern, (a) $17 \mathrm{y}$, 우, r, m; ws shows a lateral movement to the right; (b) same participant as in 8(a), 1, m; ws shows a lateral movement to the left, but also to the right $(\rightarrow)$; (c) $52 \mathrm{y}$, 우, $\mathrm{r}$ h; ws shows a lateral movement to the left (Class III with cross bite); (d) 49y, ô, r, s, ws with a triangular movement pattern, the arrows indicate the dynamics.
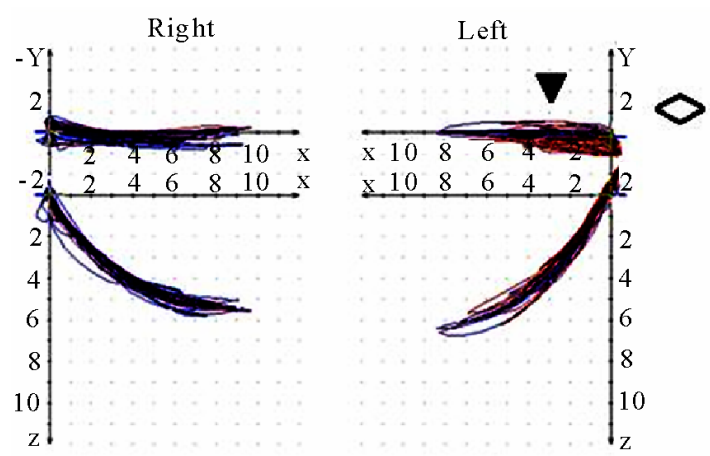

(b)
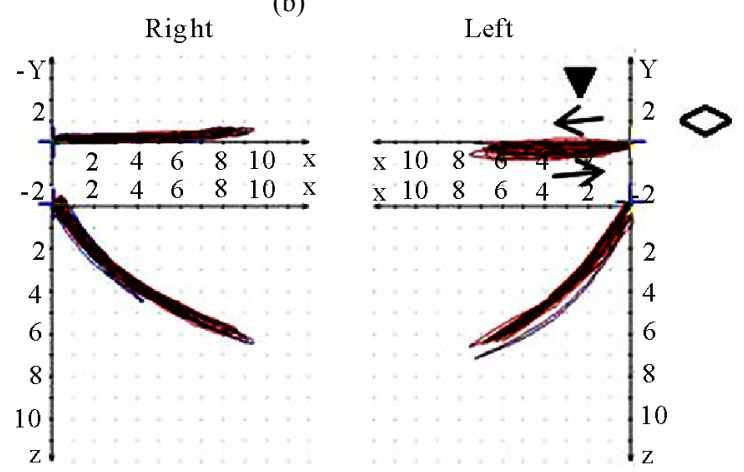

(d)

Figure 9. Coronar view $(\diamond)$ of recorded mastication pattern, (a) 17y,, , r, m; ws shows $(\boldsymbol{\nabla})$ shows a distinct lateral movement to the right; (b) same participant as in $9(\mathrm{a}), 1, \mathrm{~m}$; ws ( $\boldsymbol{\nabla})$ plots movements to both sides; (c) $21 \mathrm{y}$, o , r, m; ws ( $\boldsymbol{\nabla}$ ) charts a movement to the right during opening and to the left during closing $(\rightarrow)$; (d) same participant as in 9 (c), 1, m; ws ( $)$ ) shows a straight movement during opening and a shift to the left while closing $(\rightarrow)$. 
nAS and demonstrate these in a similar fashion as an ECG. A single masticatory movement is represented by a peak. The frequency and harmony of a masticatory movement can be viewed separately over an entire mastication process (18 seconds). The height, breadth and symmetry (steepness of the phase before the peak to the steepness of the phase after the peak) of individual peaks can be described. From such visualization one would basically expect a uniform time-pathway diagram with regular peaks, which reduce in height as the model food is minced and broken up. However, this is a speculative assumption and is not confirmed by our perusal of the published literature. Nevertheless, the time-pathway diagram of mastication can be described and analyzed by this method (Figure 10).

Interesting data can be derived from the translation-rotation diagram. Movements can be divided into two components, namely translation (horizontal axis) and rotation (vertical axis), and visualized accordingly. Characteristic differences seem to exist between AS and nAS: while AS is associated with greater rotation, nAS is marked by significant translation (Figure 11). In this recording, the accuracy of movements close to centric occlusion during mastication can be analyzed well. These sections of movement, which are always accompanied by deceleration, impose great demands on the neuromuscular system. The position and precision of these sections of motion should be included in the investigation procedure. The harmony of motions can be analyzed particularly well in this type of visualization. The direction of rotation of the pathways thus recorded may be significant. Clockwise and anti-clockwise movements

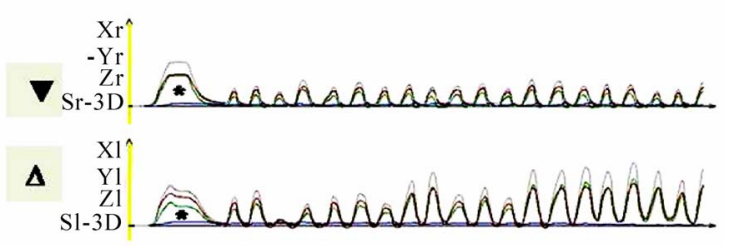

(a)

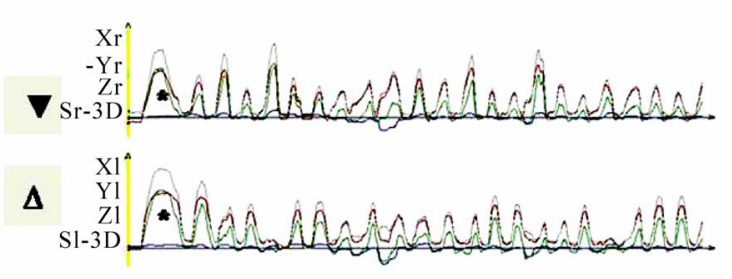

(c) are possible; AS and nAS may be oriented in different directions. A change in the direction of rotation may be observed in some cases. However, a conclusive statement about the potential pathological significance of such dynamic features cannot be made at the present time (Figure 12).

Gibbs and co-workers as well as other authors have described masticatory movement on the basis of the motion of specific occlusal points such as the points of the mandibular molars or the incisal edge of the lower front teeth [5,6]. Similar visualizations with the aid of CADIAX diagnostic software permit simulation of movements in individual occlusal regions. Sagittal, frontal and coronary views permit spatial analysis. The extent of motion and the direction of motion on the laterotrusion side (i.e., AS) and the mediotrusion side (i.e., nAS) can be analyzed. Notably, the direction of motion of the closing movement is not from the outer lower aspect to the upper inner aspect-in the manner described by Gibbs and co-workers. It was found that the dynamics of some masticatory cycles are changed, and the AS now serves as the mediotrusion side while the food bolus is retained on the same side (Figure 13).

Obviously, the above mentioned analyses must always be viewed with regard to the different degrees of hardness of the SFM. The load imposed on the stomatognathic system during mastication of hard food necessarily alters the course of the motion. In a fully functioning masticatory organ, this additional load should be of no major importance. The effects of such loads may be more pronounced in the presence of functional disorders and/or deviations in occlusion (Figure 14).

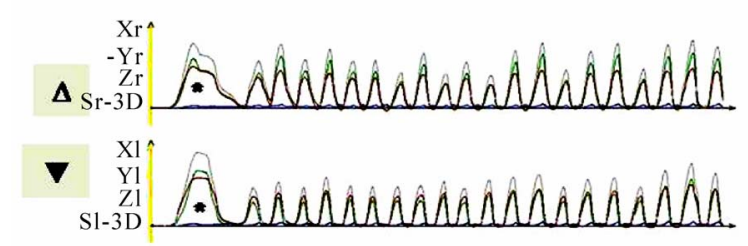

(b)

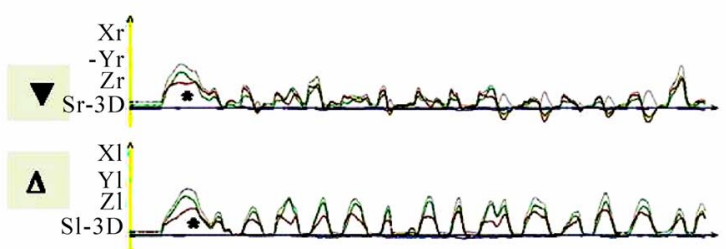

(d)

Figure 10. Time-distance diagram of recorded chewing pattern, (a) $21 \mathrm{y}, q, \mathrm{r}, \mathrm{s}$; subsequent to the initial longer and wider mouth opening $(*)$ due to food intake, the ws $(\boldsymbol{\nabla})$ plots harmonic and constant chewing cycles. The nws $(\triangle)$ reveals an active positioning of the mandible due to the unequal height of the peaks; (b) same participant as in 9(a), 1, s; subsequent to the initial longer and wider mouth opening $(*)$ due to food intake, the ws $(\boldsymbol{\nabla})$ plots harmonic and constant chewing cycles. The nws $(\triangle)$ shows an active positioning of the mandible due to the unequal height of the peaks; (c) 51y, $\widehat{\jmath}, \mathrm{r}, \mathrm{h}$; chewing cycles are irregular; (d) $52 \mathrm{y}, \widehat{\partial}, \mathrm{r}, \mathrm{s}$; the loss of harmony and constancy of chewing cycles is obvious. 


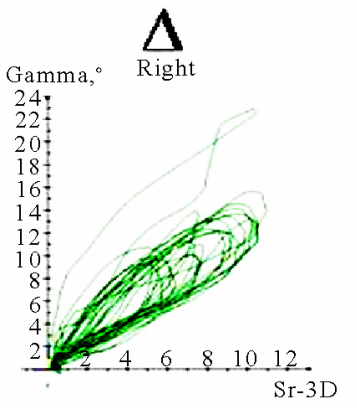

(a)
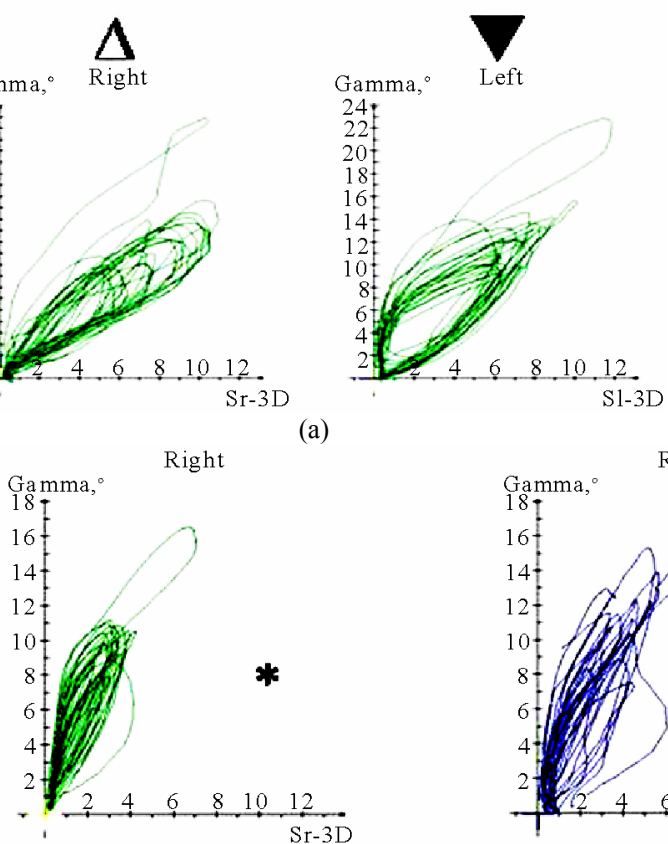

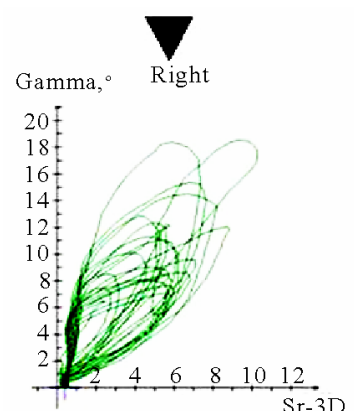

(b)

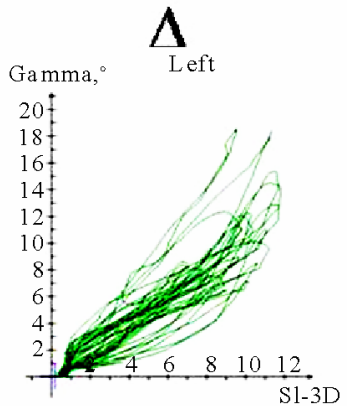

Right
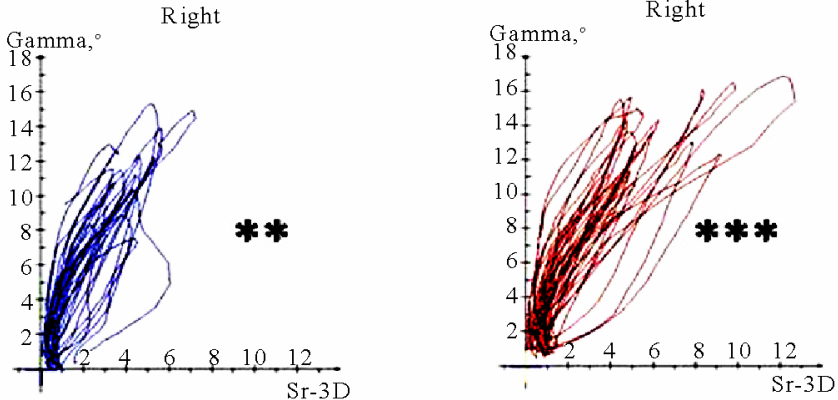

(c)

Figure 11. Translation-rotations curves of recorded chewing pattern (rotation is traced vertical, translation horizontal), (a) 21y, $+, 1, \mathrm{~s}$; the rotation on the ws $(\boldsymbol{\nabla})$ is larger then on the nws $(\triangle)$; (b) 18y, ô, r, s; the rotation on the ws $(\boldsymbol{\nabla})$ is larger then on the nws $(\triangle)$; the difference is more distinct compared to $11(\mathrm{a})$; (c) $21 \mathrm{y}, \mathrm{O}, \mathrm{r}, \mathrm{s}(*), \mathrm{m}(* *), \mathrm{h}(* * *)$; only the ws is displayed. The load and additional needed movements can be observed when chewing soft $(*)$, medium $(* *)$ of hard $(* * *)$ standard food.
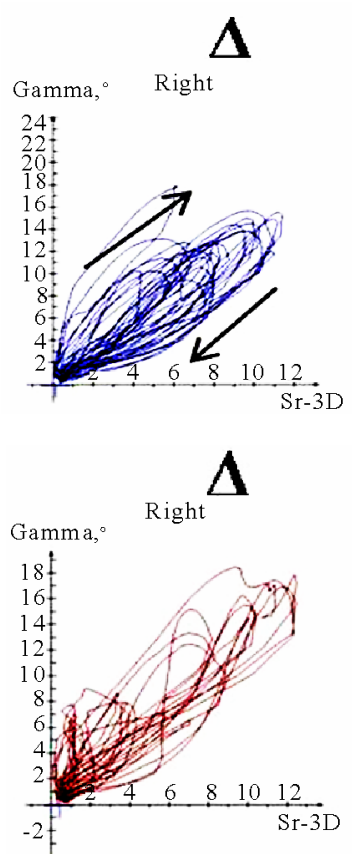

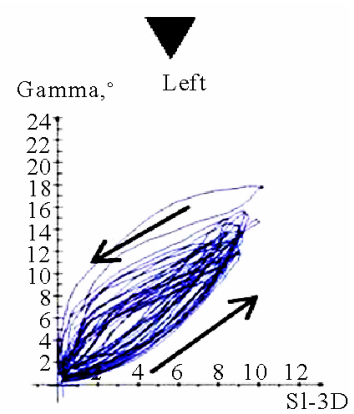

(a)

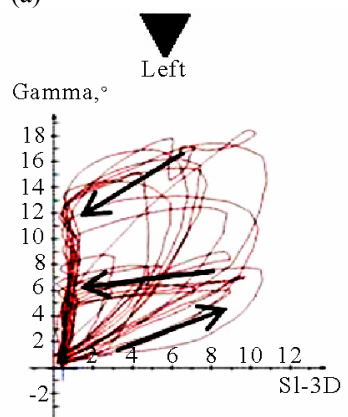

(c)

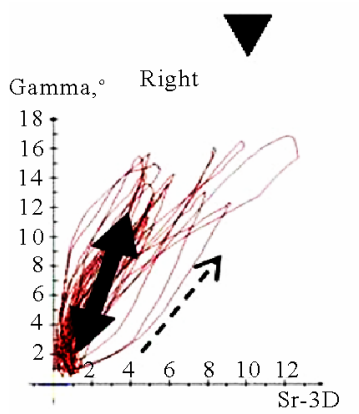

(b)
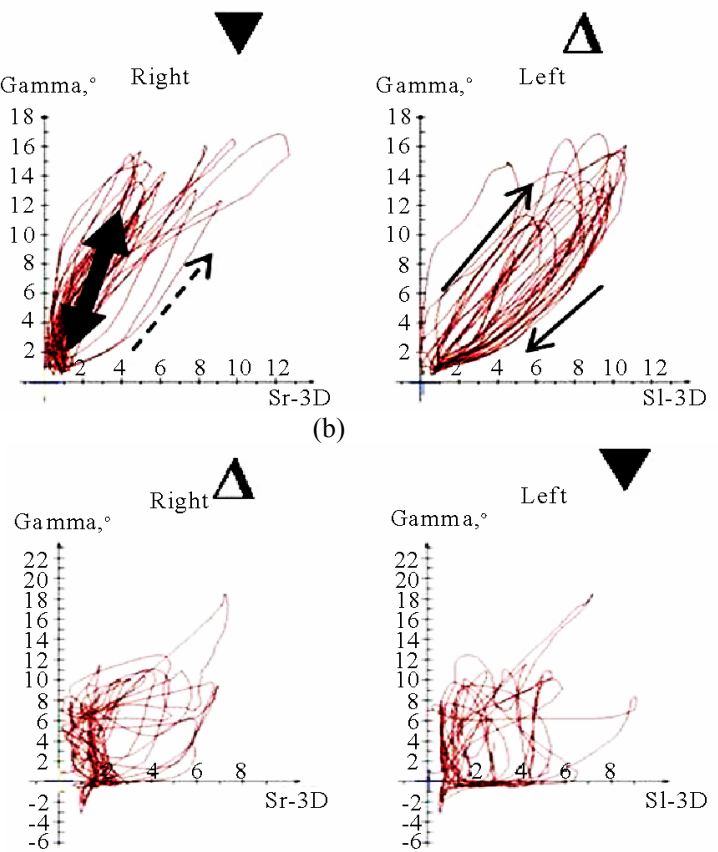

(d)

Figure 12. Translation-Rotations curves of recorded chewing pattern, (a) 21a,, , 1, m; the ws ( $\mathbf{\nabla}$ ) plots a counterclockwise direction of rotation, while the nws $(\triangle)$ displays a clockwise rotation pattern; (b) same participant as in 12(a), r, h; the ws $(\boldsymbol{\nabla})$ do not show a particular rotation pattern $(\longleftrightarrow \longrightarrow)$, only the final chewing cycles are executed with a counter clockwise rotation $(\longrightarrow)$. The nws $(\triangle)$ plots a clockwise rotation; (c) 17(a),, , l, h; the ws ( $\boldsymbol{\nabla})$ displays a counterclockwise direction of rotation, but to an unequal extend. The nws $(\triangle$ ) plots an indifferent rotation pattern; (d) 52a,, , 1, h; ws $(\boldsymbol{\nabla})$, but also nws $(\triangle)$ shows an undetermined rotation behavior. 


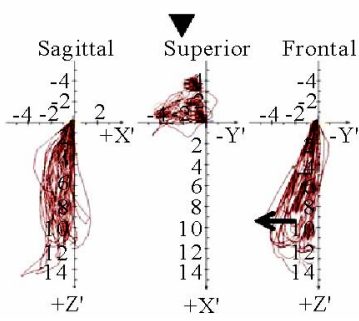

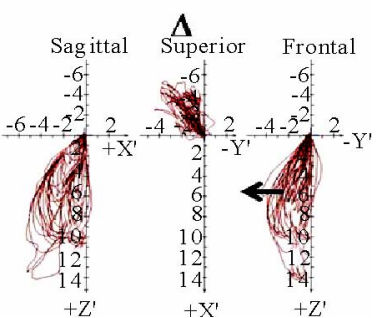

(a)

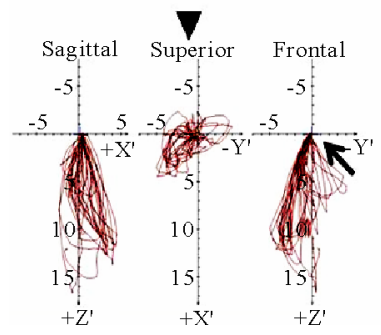

(b)

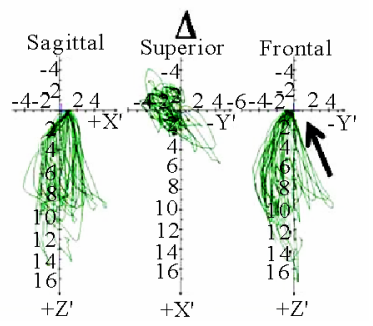

Figure 13. Tooth movements (Coordinates ws $(\nabla) x=30, y=30, z=30$; coordinates nws $(\triangle) x=30, y=-30, z=30$ ), (a) 18a, $\widehat{\partial}$, r, h; ws $(\boldsymbol{\nabla})$ and nws $(\triangle)$ show a lateral movement in direction to the ws $(\leftarrow)$. The sagittal view demonstrates the movement back and downward (participant with dental Class 1); (b) 18a, $\widehat{\partial}, \mathrm{r}, \mathrm{m}$; the frontal view reveals a similar movement, but some chewing strokes are conducted in an atypical manner from down and left $(\uparrow)$. The sagittal movement is more oriented down and anterior (participant with Class 11/1).
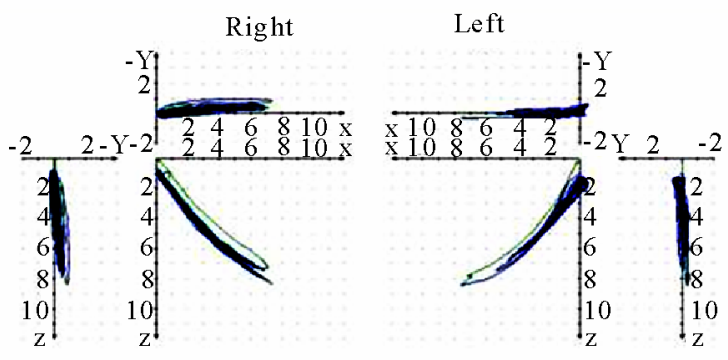

(a)
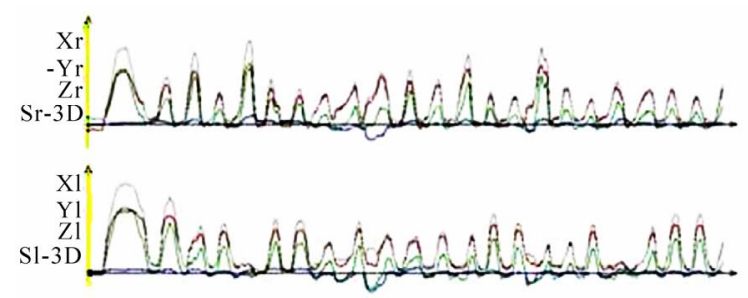

(b)
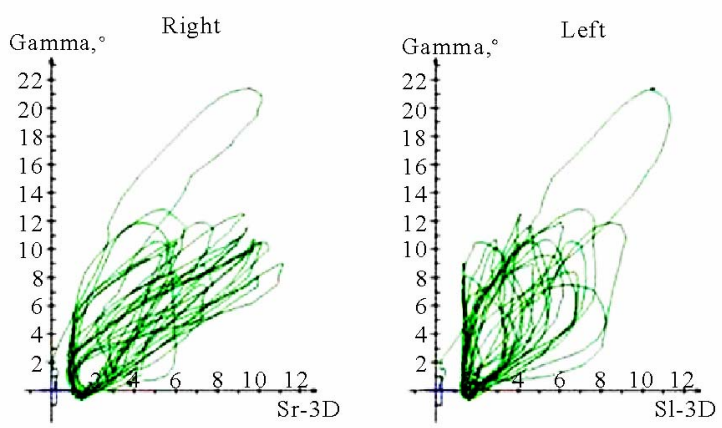

(c)
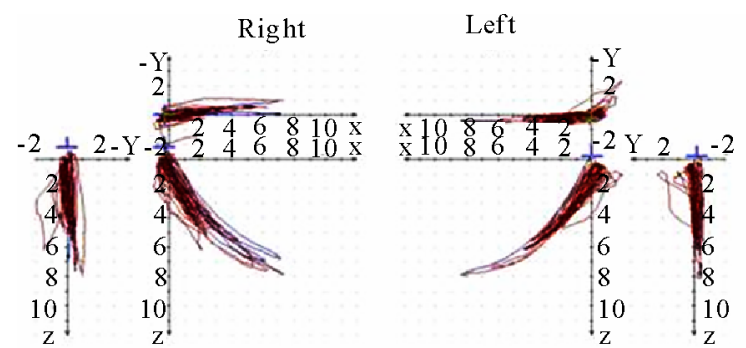

(d)
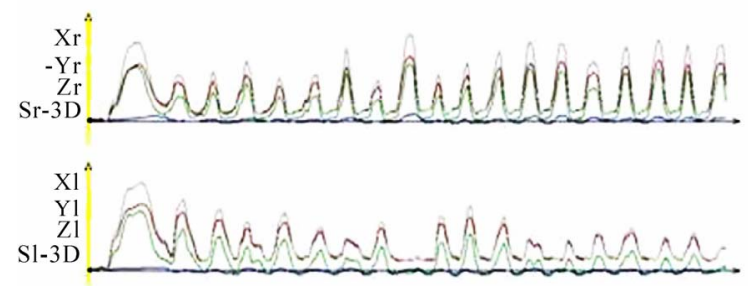

(e)
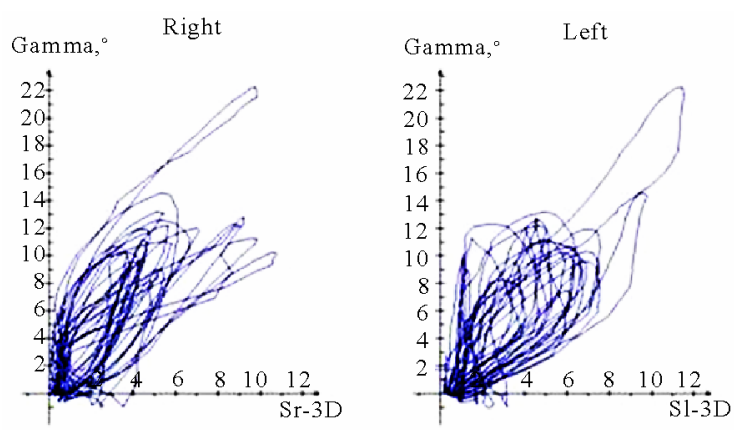

(f)

Figure 14. Demonstration of the loading of the chewing organ due to the different hardness of the model food, (a, b, c) 51a, m, $\mathrm{r}, \mathrm{s} ;(\mathrm{b}, \mathrm{d}, \mathrm{f})$ same participant as in 14(a), $\mathrm{r}, \mathrm{h}$.

Bilateral mastication provides no significant additional information for quantitative and qualitative descriptions. However, due to the harmony of changing sides, one may draw conclusions about coordination with other muscle groups such as the floor of the mouth, the suprahyoid muscles, the postural muscles, the tongue, and the mimic muscles (Figure 15). One should consider the fact that especially shifting the food bolus from 


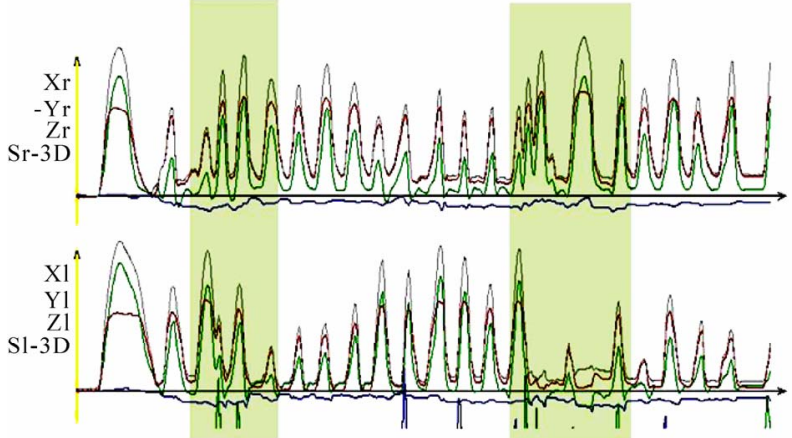

Figure 15. Time-distance diagram of a chewing sequence, up right side, down left side. The participant was asked to change the chewing side twice (shaded areas). The change in the regular chewing cycles can be seen clearly.

the right to the left side or vice versa is rendered much more difficult by the paraocclusal clutch and its handle. The usual physiological processes of the muscles of the cheek, the tongue, and especially the lips are impaired.

During and after the recording of masticatory movements, the proband should always be asked about any sensations or side effects that may have occurred. It was found that the nine recordings of masticatory movements do impose a load on the masticatory muscles and the craniomandibular joints. Even asymptomatic probands notice the loads and also perceive certain muscular sensations (general fatigue of muscles, individual sites of pain in muscles, pain in the craniomandibular joint either spontaneously or on palpation).

\section{SUMMARY AND CONCLUSIONS}

This article highlights the significance of a standardized procedure for analysis of human mastication. A database consequently created should, in the future, enable the dentist to comprehend and describe the physiological masticatory pattern and all of its conceivable individual variations. Based on the physiological processes thus identified, it will be possible to determine parameters that influence mastication and utilize these for the individual analysis. The discovery of deviations from the physiological masticatory pattern, and especially the identification of pathophysiological masticatory movements by this process, might pave the way for new strategic approaches in the diagnosis and treatment of the stomatognathic system. However, dynamic processes can only be analyzed on the basis of standardization of the data thus obtained. Therefore, this article is intended to serve as a contribution to the discussion and may initiate new research approaches in the clinical as well as experimental setting.

\section{ACKNOWLEDGEMENTS}

The authors declare no conflict of interest.

\section{REFERENCES}

[1] Geissler, C.A. and Bates, J.F. (1984) The nutritional effects of tooth loss. American Society for Clinical Nutrition, 39(3), 478-489.

[2] Ritchie, C.S., Joshipura, K., Hung, H.-C. and Douglass, C.W. (2002) Nutrition as a mediator in the relation between oral and systemic disease: Associations between specific measures of adult oral health and nutrition outcomes. Critical Reviews in Oral Biology and Medicine, 13(3), 291-300.

[3] Dienel, M. (2006) Ein neu entdeckter Schutzfaktor vor Alzheimer-Demenz: Zahlreiche noch erhaltene Zähne-die Neuburger Demenzstudie. European Journal of Geriatrics, 8(3), 166-170.

[4] Felicio, C.M., De Melchior, M.O., Da Silva, M.A. and Celeghini, R.M.S. (2007) Masticatory performance in adults related to temporo-mandibular disorder and dental occlusion. Pró-Fono Revista de Atualização Cientifica, 19(2), 151-158.

[5] Lassauzay, C., Peyron, M.A., Albuisson, E., Dransfield, E. and Woda, A. (2000) Variability of the masticatory process during chewing of elastic model foods. European Journal of Oral Sciences, 108(6), 484-492.

[6] Peyron, M.A., Blanc, O., Lund, J.P. and Woda, A. (2003) Influence of Age on adaptability of human mastication. Journal of Neurophysiology, 92(2), 773-779.

[7] Foster, K.D., Woda, A. and Peyron, M.A. (2006) Effect of texture of plastic and elastic model foods on the parameters of mastication. Journal of Neurophysiology, 95(6), 3469-3479.

[8] Schneider, G. and Sender, B. (2002) Clinical relevance of a simple fragmentation model to evaluate human masticatory performance. Journal of Oral Rehabilitation, 29(8), 731-736.

[9] Sasaguri, K., Sato, S., Hirano, Y., et al. (2004) Involvement of chewing in memory processes in humans: an approach using fMRI. International Congress Series, 1270, 111-116

[10] Huddleston Slater, J.J., Visscher, C.M., Lobbezoo, F. and Naeije, M. (1999) The intra-articular distance within the TMJ during free and loaded closing movements. Journal of Dental Research, 78(12), 1815-1820.

[11] Naeije, M. and Hofman, N. (2003) Biomechanics of the human Temporomandibular Joint during chewing. Journal of Dental Research, 82(7), 528-531.

[12] Gibbs, C.H., Mahan, P.E., Lundeen, H.C., et al. (1981) Occlusal forces during chewing-Influences of biting strength and food consistency. Journal of Prosthetic Dentistry, 46(5), 561-567.

[13] Proeschel, P.A. and Raum, J. (2003) Task-dependence of jaw elevator and depressor co-activation. Journal of Dental Research, 82(8), 617-620.

[14] Proeschel, P.A. and Morneburg, T. (2002) Task-dependence of activity/bite force relations and its impact on estimation of chewing force from EMG. Journal of Dental Research, 81(7), 464-468.

[15] Peyron, M.A., Lassauzay, C. and Woda, A. (2002) Effects of increased hardness on jaw movement and muscle ac- 
tivity during chewing of visco-elastic model foods. Experimental Brain Research, 142(1), 41-51.

[16] Gibbs, C.H., Wickwire, N.A., Jacobson, A.P., Lundeen, H.C., Mahan, P.E. and Lupkiewicz, S.M. (1982) Comparison of typical chewing patterns in normal children and adults. Journal of American Dental Association, 105(1), 33-42.

[17] Miyawaki, S., Ohkochi, N., Kawakami, T. and Sugimura, M. (2000) Effect of food size on the movement of the mandibular first molars and condyles during deliberate unilateral mastication in humans. Journal of Dental Research, 79(7), 1525-1531.

[18] Pereira, L.J., Gavião, M.B., Engelen, L. and Van der Bilt, A. (2007) Mastication and swallowing: Influence of fluid addition to foods. Journal of Applied Oral Science, 15(1), 55-60.
[19] Slavicek, G., Soiher, M., Gruber, H., Siegl, P. and Oxtoby, M. (2009) A novel standard food model to analyze the individual parameters of human mastication. International Journal of Stomatology and Occlusion Medicine, 2(4), 163-174.

[20] Slavicek, G., Soiher, M., Gruber, H., Siegl, P. and Oxtoby, M. (2009) Fallstudien zur analyse des Kauens Teil 1: Die standardanalyse. Stomatologie, 106(7), 119-129.

[21] Slavicek, G., Soiher, M., Gruber, H., Siegl, P. and Oxtoby, M. (2009) Fallstudien zur analyse des Kauens Teil 2: Spezielle analysemöglichkeiten. Stomatologie, 106(8), 137-148.

[22] Slavicek, G., Soiher, M., Gruber, H., Siegl, P. and Oxtoby, M. (2010) Fallstudien zur analyse des Kauens Teil 3: Analyse von Höckerbewegungen. Stomatologie, 107(1), $1-7$. 